\title{
Millivolt Squared per Hertz
}

National Cancer Institute

\section{Source}

National Cancer Institute. Millivolt Squared per Hertz. NCI Thesaurus. Code C114241.

A unit equal to one thousandth of a volt squared per unit of frequency equal to one

Hertz. 